\title{
A fourth order non-polynomial quintic spline collocation technique for solving time fractional superdiffusion equations
}

\author{
Muhammad Amin ${ }^{1}$, Muhammad Abbas ${ }^{2 *}$ (D), Muhammad Kashif lqbal ${ }^{3}$, Ahmad Izani Md. Ismail ${ }^{4}$ and \\ Dumitru Baleanu $u^{5,6,7}$
}

"Correspondence:
muhammad.abbas@uos.edu.pk
2Department of Mathematics,
University of Sargodha, Sargodha,
Pakistan
Full list of author information is
available at the end of the article

\begin{abstract}
The purpose of this article is to present a technique for the numerical solution of Caputo time fractional superdiffusion equation. The central difference approximation is used to discretize the time derivative, while non-polynomial quintic spline is employed as an interpolating function in the spatial direction. The proposed method is shown to be unconditionally stable and $O\left(h^{4}+\Delta t^{2}\right)$ accurate. In order to check the feasibility of the proposed technique, some test examples have been considered and the simulation results are compared with those available in the existing literature.
\end{abstract}

Keywords: Non-polynomial quintic spline; Finite central difference approach; Superdiffusion equation; Caputo time fractional derivative

\section{Introduction}

In this article, we consider the following time fractional fourth order superdiffusion equation [1]:

$$
\frac{\partial^{\alpha} y}{\partial t^{\alpha}}+\gamma \frac{\partial^{4} y}{\partial z^{4}}=f(z, t), \quad 0 \leq z \leq L, 0 \leq t \leq T,
$$

with the initial/end conditions

$$
\begin{aligned}
& y(z, 0)=\phi(z), \quad y_{t}(z, 0)=\psi(z), \\
& y(0, t)=y(L, t)=0, \\
& y_{z z}(0, t)=y_{z z}(L, t)=0,
\end{aligned}
$$

where $\alpha \in(1,2]$ denotes the order of time fractional derivative, $\gamma$ is a constant, and $\phi(z)$ is continuous on $[0, L]$.

Fourth order time fractional partial differential equations (PDEs) arise in mathematical modeling of several plate-like objects [2]. Most of the analytical techniques for solving fractional order PDEs are based on Laplace and Fourier transforms, while others involve the separation of variables technique $[3,4]$. Some semi-analytic methods have also

(c) The Author(s) 2019. This article is licensed under a Creative Commons Attribution 4.0 International License, which permits use, sharing, adaptation, distribution and reproduction in any medium or format, as long as you give appropriate credit to the original author(s) and the source, provide a link to the Creative Commons licence, and indicate if changes were made. The images or other third party material in this article are included in the article's Creative Commons licence, unless indicated otherwise in a credit line to the material. If material is not included in the article's Creative Commons licence and your intended use is not permitted by statutory regulation or exceeds the permitted use, you will need to obtain permission directly from the copyright holder. To view a copy of this licence, visit http://creativecommons.org/licenses/by/4.0/. 
been employed to explore series solution for fractional order PDEs. These included homotopy analysis method [5], Adomian decomposition method [6, 7], homotopy perturbation method [8], variational iteration method [9], and fractional differential transformation method [10].

In recent years, spline functions have also been frequently employed for the numerical solution of fractional order PDEs. These functions have advantages over the usual finite difference methods as they can provide a continuous differentiable approximation to the unknown function in the spatial domain with good accuracy. The simple and straightforward application of spline functions provides enough motivation to employ them for the numerical study of fractional PDEs. Zahra and Elkholy [11] employed cubic spline functions combined with shooting method for solving fractional Bagley-Torvik equation. Talaat and Danaf [12] applied the quadratic spline method for numerical investigation of fractional diffusion equation. Siddiqi and Arshed [13] used the quintic B-spline collocation method for numerical solution of time fractional fourth order PDEs. In [14], the authors introduced new fractional order spline functions to study the numerical solution of fractional Bagely-Torvik equation. Mohyu-Din et al. [15] investigated the extended Bspline solution of time fractional advection diffusion equation by means of a fully implicit finite difference scheme. Li et al. [16] developed a non-polynomial spline scheme to solve time fractional nonlinear Schrodinger equation. In [17], Pezza and Pitolli used a fractional spline collocation Galerkin scheme to develop series solution for time fractional diffusion equation. Khalid et al. [18] utilized the non-polynomial quintic spline collocation method to explore the numerical solution of fourth order fractional boundary value problem, involving product terms. In [19], Amin et al. employed the quintic non-polynomial spline collocation scheme for solving time fractional fourth order PDEs.

There are several techniques to deal with the fractional differentiation but RiemannLiouville's and Caputo's approaches are the most common. Here, we utilize Caputo's definition as it allows us to use the ordinary initial/boundary constraints. The Caputo time fractional derivative of order $\alpha$ is expressed as

$$
\frac{\partial^{\alpha} y(z, t)}{\partial t^{\alpha}}= \begin{cases}\frac{1}{\Gamma(2-\alpha)} \int_{0}^{t} \frac{\partial^{2} y(z, s)}{\partial s^{2}} \frac{d s}{(t-s)^{\alpha}-1}, & 1<\alpha<2, \\ \frac{\partial^{2} y(z, t)}{\partial t^{2}}, & \alpha=2 .\end{cases}
$$

This paper aims to develop a spline collocation approach for numerical solution of fourth order time fractional superdiffusion problem. For spatial discretization, a non-polynomial quintic spline interpolant, composed of trigonometric and polynomial components, is used. For temporal discretization, a central difference approximation is used.

The outline of this paper is as follows: A short description of the non-polynomial quintic spline technique is given in Sect. 2. The consistency relations between the spline approximation and its spatial derivatives at the grid points are derived in this section. In Sect. 3, the application of Caputo's definition and finite central difference formulation for temporal discretization is shown. The stability and convergence of the proposed problem is discussed in Sect. 4. The discretization in space direction is given in Sect. 5. The approximate results are discussed in Sect. 6, and the conclusion of the proposed study is given in Sect. 7.

\section{Non-polynomial quintic spline functions}

In this section, we construct the formulation and derive the truncation error of nonpolynomial quintic spline functions. 


\subsection{Formulation}

Let $z_{i}=a+i h$ be the grid points of a uniform partition of [0,L] dividing it into the subintervals $\left[z_{i}, z_{i+1}\right]$, where $h=\frac{L}{N}$ and $0 \leq i \leq N$. We consider that $y(z)$ is sufficiently differentiable in $[0, L]$ and $Y(z)$ is its quintic non-polynomial spline approximation. Let each non-polynomial spline segment $S_{i}(z)$ have the following form $[18,19]$ :

$$
\begin{aligned}
S_{i}(z)= & a_{i} \cos \eta\left(z-z_{i}\right)+b_{i} \sin \eta\left(z-z_{i}\right)+c_{i}\left(z-z_{i}\right)^{3} \\
& +d_{i}\left(z-z_{i}\right)^{2}+e_{i}\left(z-z_{i}\right)+f_{i}, \quad 0 \leq i \leq N
\end{aligned}
$$

where $a_{i}, b_{i}, c_{i}, d_{i}, e_{i}$, and $f_{i}$ are constants to be determined and $\eta$ denotes the frequency of the trigonometric functions. Moreover,

$$
S_{i}(z) \in C^{\infty}[0, L]
$$

and

$$
Y(z)=S_{i}(z), \quad \forall z \in\left[z_{i}, z_{i+1}\right], i=0,1,2, \ldots, N .
$$

Let

$$
Y_{i}=S_{i}\left(z_{i}\right), \quad M_{i}=Y^{\prime \prime}\left(z_{i}\right), \quad F_{i}=Y^{(4)}\left(z_{i}\right)
$$

The constants involved in $S_{i}(z)$ can be expressed as follows:

$$
\begin{aligned}
a_{i} & =\frac{h^{4}}{\theta^{4}} F_{i}, \\
b_{i} & =\frac{h^{4}}{\theta^{4} \sin (\theta)}\left(F_{i+1}-F_{i} \cos (\theta)\right), \\
c_{i} & =\frac{1}{6 h}\left(M_{i+1}-M_{i}\right)+\frac{h}{6 \theta^{2}}\left(F_{i+1}-F_{i}\right), \\
d_{i} & =\frac{1}{2} M_{i}+\frac{h^{2}}{2 \theta^{2}} F_{i}, \\
e_{i} & =\frac{1}{h}\left(Y_{i+1}-Y_{i}\right)+\left(\frac{h^{3}}{\theta^{4}}-\frac{h^{3}}{3 \theta^{2}}\right) F_{i}-\left(\frac{h^{3}}{\theta^{4}},+\frac{h^{3}}{6 \theta^{2}}\right) F_{i+1}-\frac{h}{6}\left(M_{i+1}+2 M_{i}\right), \\
f_{i} & =Y_{i}-\frac{h^{4}}{\theta^{4}} F_{i},
\end{aligned}
$$

where $\theta=\eta h$ and $i=0 \leq i \leq N$. Using 1st and 3rd derivative continuities at the knots, $S_{i-1}^{(\tau)}\left(z_{i}\right)=S_{i}^{(\tau)}\left(z_{i}\right)$ for $\tau=1,3$, the following important relations can be derived:

$$
\begin{aligned}
M_{i+1}+4 M_{i}+M_{i-1}= & \frac{6}{h^{2}}\left(Y_{i+1}-2 Y_{i}+Y_{i-1}\right)+\frac{6 h^{2}}{\theta^{2}}\left(\frac{1}{\theta \sin (\theta)}-\frac{1}{\theta^{2}}-\frac{1}{6}\right)\left(F_{i+1}+F_{i-1}\right) \\
& +\frac{6 h^{2}}{\theta^{2}}\left(\frac{2}{\theta^{2}}-\frac{2 \cos (\theta)}{\theta \sin (\theta)}-\frac{4}{6}\right) F_{i}
\end{aligned}
$$

and

$$
M_{i+1}-2 M_{i}+M_{i-1}=h^{2}\left(\frac{1}{\theta \sin (\theta)}-\frac{1}{\theta^{2}}\right)\left(F_{i+1}+F_{i-1}\right)+2 h^{2}\left(\frac{1}{\theta^{2}}-\frac{\cos (\theta)}{\theta \sin (\theta)}\right) F_{i} .
$$


Solving (4) and (5) yields

$$
\begin{aligned}
M_{i}= & \frac{1}{h^{2}}\left(Y_{i+1}-2 Y_{i}+Y_{i-1}\right)+h^{2}\left(\frac{1}{\theta^{3} \sin (\theta)}-\frac{1}{6 \theta \sin (\theta)}-\frac{1}{\theta^{4}}\right)\left(F_{i+1}+F_{i-1}\right) . \\
& +h^{2}\left(\frac{2}{\theta^{4}}-\frac{2 \cos (\theta)}{\theta^{3} \sin (\theta)}+\frac{2 \cos (\theta)}{6 \theta \sin (\theta)}-\frac{1}{\theta^{2}}\right)\left(F_{i}\right) .
\end{aligned}
$$

Using (5) and (6), we obtain the following consistency relation involving $F_{i}$ and $Y_{i}$ for $i=$ $2,3, \ldots, N-2$ :

$$
Y_{i+2}-4 Y_{i+1}+6 Y_{i}-4 Y_{i-1}+Y_{i-2}=h^{4}\left(\mu_{1} F_{i-2}+v_{1} F_{i-1}+\kappa_{1} F_{i}+v_{1} F_{i+1}+\mu_{1} F_{i+2}\right),
$$

where

$$
\begin{aligned}
& \mu_{1}=\frac{1}{\theta^{4}}+\frac{1}{6 \theta \sin (\theta)}-\frac{1}{\theta^{3} \sin (\theta)}, \quad \nu_{1}=\frac{2+2 \cos (\theta)}{\theta^{3} \sin (\theta)}+\frac{2-\cos (\theta)}{3 \theta \sin (\theta)}-\frac{4}{\theta^{4}}, \\
& \kappa_{1}=\frac{1-4 \cos (\theta)}{3 \theta \sin (\theta)}-\frac{2+4 \cos (\theta)}{\theta^{3} \sin (\theta)}+\frac{6}{\theta^{4}} .
\end{aligned}
$$

Relation (7) produces $(N-3)$ algebraic equations involving $(N-1)$ unknowns, $Y_{i}, i=$ $1,2, \ldots, N-1$. In order to solve this system, we obtain two more conditions as follows:

Setting $i=1,2$ in (4), we have

$$
M_{0}+4 M_{1}+M_{2}=\frac{6}{h^{2}}\left(Y_{0}-2 Y_{1}+Y_{2}\right)+\tilde{\tau}\left(F_{0}+F_{2}\right)+\tilde{\kappa} F_{1}
$$

and

$$
M_{1}+4 M_{2}+M_{3}=\frac{6}{h^{2}}\left(Y_{1}-2 Y_{2}+Y_{3}\right)+\tilde{\tau}\left(F_{1}+F_{3}\right)+\tilde{\kappa} F_{2} .
$$

Similarly, using (5), the following two expressions can be derived with $i=1,2$ :

$$
M_{0}-2 M_{1}+M_{2}=\widetilde{\widetilde{\tau}}\left(F_{0}+F_{2}\right)+\widetilde{\widetilde{\kappa}} F_{1}
$$

and

$$
M_{1}-2 M_{2}+M_{3}=\widetilde{\widetilde{\tau}}\left(F_{1}+F_{3}\right)+\widetilde{\widetilde{\kappa}} F_{2}
$$

where

$$
\begin{aligned}
& \tilde{\tau}=\frac{6 h^{2}}{\theta^{2}}\left(\frac{1}{\theta \sin \theta}-\frac{1}{\theta^{2}}-\frac{1}{6}\right), \quad \tilde{\kappa}=\frac{6 h^{2}}{\theta^{2}}\left(\frac{2}{\theta^{2}}-\frac{2 \cos (\theta)}{\theta \sin (\theta)}-\frac{4}{6}\right), \\
& \tilde{\tau}=h^{2}\left(\frac{1}{\theta \sin (\theta)}-\frac{1}{\theta^{2}}\right) \quad \text { and } \quad \tilde{\widetilde{\kappa}}=2 h^{2}\left(\frac{1}{\theta^{2}}-\frac{\cos (\theta)}{\theta \sin (\theta)}\right) .
\end{aligned}
$$

From (8) and (10), we have

$$
M_{1}=\frac{1}{h^{2}}\left(Y_{0}-2 Y_{1}+Y_{2}\right)+\frac{\tilde{\tau}-\widetilde{\tau}}{6}\left(F_{0}+F_{2}\right)+\frac{\tilde{\kappa}-\widetilde{\widetilde{\kappa}}}{6} F_{1} .
$$


Similarly, using (9) and (11), we obtain

$$
M_{2}=\frac{1}{h^{2}}\left(Y_{1}-2 Y_{2}+Y_{3}\right)+\frac{\tilde{\tau}-\tilde{\tau}}{6}\left(F_{1}+F_{3}\right)+\frac{\tilde{\kappa}-\tilde{\kappa}}{6} F_{2} .
$$

Substituting (12) and (13) into (8) for $i=1$ yields

$$
2 Y_{0}-5 Y_{1}+4 Y_{2}-Y_{3}=h^{2} M_{0}-h^{4}\left(\varpi_{0} F_{0}+\varpi_{1} F_{1}+\varpi_{2} F_{2}+\varpi_{3} F_{3}\right) .
$$

Also, for $i=n$, the following relation can be established:

$$
\begin{aligned}
& Y_{N-3}-4 Y_{N-2}+5 Y_{N-1}-2 Y_{N} \\
& \quad=-h^{2} M_{N}+h^{4}\left(\varpi_{3} F_{N-3}+\varpi_{2} F_{N-2}+\varpi_{1} F_{N-1}+\varpi_{0} F_{N}\right),
\end{aligned}
$$

where

$$
\begin{array}{ll}
\varpi_{0}=\frac{2}{\theta^{3} \sin (\theta)}-\frac{2}{\theta^{4}}+\frac{4}{6 \theta \sin (\theta)}-\frac{1}{\theta^{2}}, & \varpi_{1}=\frac{1-8 \cos (\theta)}{6 \theta \sin (\theta)}-\frac{1+4 \cos (\theta)}{\theta^{3} \sin (\theta)}+\frac{5}{\theta^{4}}, \\
\varpi_{2}=\frac{2+2 \cos (\theta)}{\theta^{3} \sin (\theta)}+\frac{2-\cos (\theta)}{3 \theta \sin (\theta)}-\frac{4}{\theta^{4}}, & \varpi_{3}=\frac{1}{6 \theta \sin (\theta)}-\frac{1}{\theta^{3} \sin (\theta)}+\frac{1}{\theta^{4}} .
\end{array}
$$

\subsection{Truncation error}

To calculate $t_{i}, 1 \leq i \leq N-1$, for the current scheme, we rewrite (7), (14), and (15) as follows:

$$
\begin{aligned}
& \tilde{t}_{1}=-2 y_{0}+5 y_{1}-4 y_{2}+y_{3}+h^{2} M_{0}-h^{4}\left(\varpi_{0} y_{0}^{(4)}+\varpi_{1} y_{1}^{(4)}+\varpi_{2} y_{2}^{(4)}+\varpi_{3} y_{3}^{(4)}\right), \\
& \tilde{t}_{i}=y_{i-2}-4 y_{i-1}+6 y_{i}-4 y_{i+1}+y_{i+2}-h^{4}\left(\alpha_{1} y_{i-2}^{(4)}+\beta_{1} y_{i-1}^{(4)}+\gamma_{1} y_{i}^{(4)}+\beta_{1} y_{i+1}^{(4)}+\alpha_{1} y_{i+2}^{(4)}\right), \\
& \tilde{t}_{N-1}=y_{N-3}-4 y_{N-2}+5 y_{N-1}-2 y_{n}+h^{2} M_{N} \\
& +h^{4}\left(\varpi_{3} y_{N-3}^{(4)}+\varpi_{2} y_{N-2}^{(4)}+\varpi_{1} y_{N-1}^{(4)}+\varpi_{0} y_{N}^{(4)}\right) .
\end{aligned}
$$

The following relations for $\tilde{t}_{i}, 1 \leq i \leq N-1$, can be established by expanding $y_{0}$, $y_{1}, y_{1}^{(4)}, y_{2}, y_{2}^{(4)}, y_{3}, y_{3}^{(4)}$, etc. about the points $z_{i}, 1 \leq i \leq N-1$, by means of Taylor's series:

$$
\tilde{t}_{i}=\left\{\begin{aligned}
&\left(\frac{11}{12}-\varpi_{0}-\varpi_{1}-\varpi_{2}-\varpi_{3}\right) h^{4} y_{i}^{(4)}+\left(\frac{1}{12}+\varpi_{0}-\varpi_{2}-2 \varpi_{3}\right) h^{5} y_{i}^{(5)} \\
&+\left(\frac{11}{90}-\frac{1}{2} \varpi_{0}-\frac{1}{2} \varpi_{2}-2 \varpi_{3}\right) h^{6} y_{i}^{(6)}+\left(\frac{1}{60}+\frac{1}{6} \varpi_{0}-\frac{1}{6} \varpi_{2}-\frac{4}{3} \varpi_{3}\right) h^{7} y_{i}^{(7)} \\
&+\left(\frac{17}{2240}-\frac{1}{24} \varpi_{0}-\frac{1}{24} \varpi_{2}-\frac{2}{3} \varpi_{3}\right) h^{8} y_{i}^{(8)}+O\left(h^{9}\right) \\
& \quad i=1 \\
&\left(1-2 \alpha_{1}-2 \beta_{1}-\gamma_{1}\right) h^{4} y_{i}^{(4)}+\left(\frac{1}{6}-4 \alpha_{1}-\beta_{1}\right) h^{6} y_{i}^{(6)} \\
&+\left(\frac{1}{180}-\frac{4}{3} \alpha_{1}-\frac{1}{12} \beta_{1}\right) h^{8} y_{i}^{(8)}+\left(\frac{17}{30240}-\frac{8}{45} \alpha_{1}-\frac{1}{360} \beta_{1}\right) h^{10} y_{i}^{(10)}+O\left(h^{11}\right) \\
& \quad i=2(1) N-2 \\
&\left(\frac{11}{12}-\varpi_{0}-\varpi_{1}-\varpi_{2}-\varpi_{3}\right) h^{4} y_{i}^{(4)}+\left(\frac{1}{12}+\varpi_{0}-\varpi_{2}-2 \varpi_{3}\right) h^{5} y_{i}^{(5)} \\
&+\left(\frac{11}{90}-\frac{1}{2} \varpi_{0}-\frac{1}{2} \varpi_{2}-2 \varpi_{3}\right) h^{6} y_{i}^{(6)}+\left(\frac{1}{60}+\frac{1}{6} \varpi_{0}-\frac{1}{6} \varpi_{2}-\frac{4}{3} \varpi_{3}\right) h^{7} y_{i}^{(7)} \\
&+\left(\frac{17}{2240}-\frac{1}{24} \varpi_{0}-\frac{1}{24} \varpi_{2}-\frac{2}{3} \varpi_{3}\right) h^{8} y_{i}^{(8)}+O\left(h^{9}\right) \\
& i=N-1 .
\end{aligned}\right.
$$


Comparing the coefficients of $y_{i}^{(\tau)}$ for $\tau=4,5,6,7$, we obtain

$$
\begin{array}{llll}
\mu_{1}=-\frac{1}{720}, & v_{1}=\frac{31}{180}, & \kappa_{1}=\frac{79}{120}, & \varpi_{0}=\frac{7}{90}, \\
\varpi_{1}=\frac{49}{72}, & \varpi_{2}=\frac{7}{45} \quad \text { and } \quad \varpi_{3}=\frac{1}{360} .
\end{array}
$$

Finally we get

$$
\tilde{t}_{i}= \begin{cases}-\frac{241}{60480} h^{8} y_{i}^{(8)}+O\left(h^{9}\right), & i=1, \\ \frac{1}{3024} h^{10} y_{i}^{(10)}+O\left(h^{11}\right), & i=2(1) N-2, \\ -\frac{241}{60480} h^{8} y_{i}^{(8)}+O\left(h^{9}\right), & i=N-1 .\end{cases}
$$

Following [19], the formulation and truncation error given in Sects. 2.1 and 2.2 respectively are reproduced here for the sake of completeness.

\section{Time discretization}

Let $t_{m}=m \Delta t$, where $\Delta t=\frac{T}{K}$ is the step size in the time direction for $m=0,1,2, \ldots, K$. To discretize the Caputo fractional time derivative at $t=t_{m+1}$, the usual central difference approach is used as follows [20]:

$$
\begin{aligned}
& \frac{\partial^{\alpha} y\left(z, t_{m+1}\right)}{\partial t^{\alpha}}=\frac{1}{\Gamma(2-\alpha)} \int_{0}^{t_{k+1}} \frac{\partial^{2} y(z, w)}{\partial w^{2}}\left(t_{m+1}-w\right)^{-\alpha+1} d w \\
& \frac{\partial^{\alpha} y\left(z, t_{m+1}\right)}{\partial t^{\alpha}} \\
& =\frac{1}{\Gamma(2-\alpha)} \sum_{k=0}^{m} \int_{t_{k}}^{t_{k+1}} \frac{\partial^{2} y(z, w)}{\partial w^{2}}\left(t_{m+1}-w\right)^{-\alpha+1} d w \\
& =\frac{1}{\Gamma(2-\alpha)} \sum_{k=0}^{m} \frac{y\left(z, t_{k+1}\right)-2 y\left(z, t_{k}\right)+y\left(z, t_{k-1}\right)}{\Delta t^{2}} \int_{t_{k}}^{t_{k+1}}\left(t_{m+1}-w\right)^{-\alpha+1} d w+l_{\Delta t}^{m+1} \\
& =\frac{1}{\Gamma(2-\alpha)} \sum_{k=0}^{m} \frac{y\left(z, t_{k+1}\right)-2 y\left(z, t_{k}\right)+y\left(z, t_{k-1}\right)}{\Delta t^{2}} \int_{t_{m-k}}^{t_{m-k+1}}(v)^{-\alpha+1} d v+l_{\Delta t}^{m+1} \\
& =\frac{1}{\Gamma(2-\alpha)} \sum_{k=0}^{m} \frac{y\left(z, t_{m-k+1}\right)-2 y\left(z, t_{m-k}\right)+y\left(z, t_{m-k-1}\right)}{\Delta t^{2}} \int_{t_{k}}^{t_{k+1}}(v)^{-\alpha} d v+l_{\Delta t}^{m+1} \\
& =\frac{1}{\Gamma(3-\alpha)} \sum_{k=0}^{m} \frac{y\left(z, t_{m-k+1}\right)-2 y\left(z, t_{m-k}\right)+y\left(z, t_{m-k-1}\right)}{\Delta t^{\alpha}}\left((k+1)^{2-\alpha}-k^{2-\alpha}\right)+l_{\Delta t}^{m+1} \\
& =\frac{1}{\Gamma(3-\alpha)} \sum_{k=0}^{m} \frac{y\left(z, t_{m-k+1}\right)-2 y\left(z, t_{m-k}\right)+y\left(z, t_{m-k-1}\right)}{\Delta t^{\alpha}}+l_{\Delta t}^{m+1},
\end{aligned}
$$

where $d_{k}=(k+1)^{2-\alpha}-k^{2-\alpha}$ and $v=\left(t_{m+1}-w\right)$.

Now, introduce a fractional differential operator $\Omega_{t}^{\alpha}$ :

$$
\Omega_{t}^{\alpha} y\left(z, t_{k+1}\right)=\frac{1}{\Gamma(3-\alpha)} \sum_{k=0}^{m} d_{k} \frac{y\left(z, t_{m-k+1}\right)-2 y\left(z, t_{m-k}\right)+y\left(z, t_{m-k-1}\right)}{\Delta t^{\alpha}} .
$$


Equation (18) can be rewritten as follows:

$$
\frac{\partial^{\alpha} y\left(z, t_{m+1}\right)}{\partial t^{\alpha}}=\Omega_{t}^{\alpha} y\left(z, t_{k+1}\right)+l_{\Delta t}^{m+1}
$$

Here, $l_{\Delta t}^{m+1}$ denotes the truncation error between $\frac{\partial^{\alpha}}{\partial t^{\alpha}} y\left(z, t_{m+1}\right)$ and $\Omega_{t}^{\alpha} y\left(z, t_{m+1}\right)$. Equation (1) can be written as

$$
\Omega_{t}^{\alpha} y\left(z, t_{m+1}\right)+\gamma \frac{\partial^{4}}{\partial z^{4}} y\left(z, t_{m+1}\right)=f\left(z, t_{m+1}\right)
$$

where $\Omega_{t}^{\alpha} y\left(z, t_{m+1}\right)$ denotes the Caputo fractional time derivative approximation at $t=$ $t_{m+1}$. Using (18), expression (20) takes the following form:

$$
\begin{aligned}
y^{m+1}(z)+\beta \gamma y_{x x x x}^{m+1}= & -d_{m} y^{-1}(z)+\left(2 d_{m}-d_{m-1}\right) y^{0}(z)+\sum_{k=1}^{m-1}\left(-d_{k-1}+2 d_{k}-d_{k+1}\right) y^{m-k}(z) \\
& +\left(2 d_{0}-d_{1}\right) y^{m}(z)+\beta f^{m+1}(z), \quad m=1,2,3, \ldots, K-1
\end{aligned}
$$

where $\beta=\Gamma(3-\alpha) \Delta t^{\alpha}$ and $y^{m+1}(z)=y\left(z, t^{m+1}\right)$ and the initial conditions are imposed as follows:

$$
\begin{aligned}
& y\left(z, t_{0}\right)=\phi(z), \quad 0 \leq z \leq L, \\
& \frac{\partial y\left(z, t_{0}\right)}{\partial t}=\psi(z), \quad 0 \leq z \leq L .
\end{aligned}
$$

Moreover, the constants $d_{k} s$ appearing in (18) possess the following properties:

- $d_{0}=1$ and $\forall k, d_{k} s>0$,

- $\left(2-d_{1}\right)-\sum_{k=1}^{m-1}\left(d_{k+1}-2 d_{k+1}+d_{k-1}\right)+\left(2 d_{m}-d_{m-1}\right)-d_{m}=1$.

The truncation error in (19) is bounded, i.e.,

$$
\left|l_{\Delta t}^{m+1}\right| \leq \zeta \Delta t^{2}
$$

Here, $\zeta$ is a constant depending on $y$.

To implement this scheme, first we calculate $y^{-1}$ as follows:

$$
\begin{aligned}
& y^{-1}(z)=y\left(z, t_{0}\right)-\Delta t y_{t}\left(z, t_{0}\right), \\
& y^{-1}(z)=\phi(z)-\Delta t \psi(z) .
\end{aligned}
$$

For $m=0,(21)$ takes the following form:

$$
y^{1}(z)+\beta \gamma y_{z z z z}^{1}=-d_{0} y^{-1}(z)+2 d_{0} y_{0}(z)+\beta f^{1}(z) .
$$

Now, Eqs. (21) and (25) together with initial/boundary conditions become a complete set of semi-discrete problem for (1).

Also, $l^{m+1}$, the error at $t=t_{m+1}$, is given by [21]

$$
l^{m+1}=\beta\left(\frac{\partial^{\alpha}}{\partial t^{\alpha}} y\left(z, t_{m+1}\right)-G_{t}^{\alpha} y\left(z, t_{m+1}\right)\right) .
$$


From Eqs. (19) and (24), the above expression can be written as

$$
\left|l^{m+1}\right|=\left|l_{\Delta t}^{m+1}\right| \leq \zeta \Delta t^{2}
$$

Some relevant functional spaces, their inner product and standard norms are defined as follows:

$$
\begin{aligned}
& \Upsilon^{2}(\eta)=\left\{u \in L^{2}(\eta), u_{z}, u_{z z} \in L^{2}(\eta)\right\}, \\
& \Upsilon_{0}^{2}(\eta)=\left\{u \in \Upsilon^{2}(\eta),\left.u\right|_{\partial \eta}=0,\left.u_{z}\right|_{\partial \eta}=0\right\}, \\
& \Upsilon^{n}(\eta)=\left\{u \in L^{2}(\eta), u_{z}^{(r)}, \forall r \leq N\right\},
\end{aligned}
$$

where $L^{2}(\eta)$ denotes the space of those measurable functions whose squares are Lebesgue integrable in $\eta$. The norm and inner product of $L^{2}(\eta)$ are given by

$$
\langle v, u\rangle=\int_{\eta} v u d z, \quad\|u\|_{0}=\langle u, u\rangle^{\frac{1}{2}}
$$

Also, for $\Upsilon^{2}(\eta)$, we take

$$
\langle v, u\rangle_{2}=\langle v, u\rangle+\left\langle v_{z}, u_{z}\right\rangle+\left\langle v_{z z}, u_{z z}\right\rangle, \quad\|u\|_{2}=\sqrt{\langle u, u\rangle_{2}}
$$

The norm $\|\cdot\|$ of the space $\Upsilon^{N}(\eta)$ is defined as

$$
\|u\|_{N}=\sqrt{\sum_{r=0}^{N}\left\|u_{x}^{(r)}\right\|_{0}^{2}}
$$

$\|\cdot\|_{2}$ is defined as

$$
\|u\|_{2}=\sqrt{\|u\|_{0}^{2}+\beta \gamma\left\|u_{x}^{(2)}\right\|_{0}^{2}}
$$

Now, to carry out the stability and convergence analysis, we need to find $y^{m+1} \in \Upsilon_{0}^{2}(\eta)$ such that $\forall u \in \Upsilon_{0}^{2}(\eta)$. From (21) and (25), we have

$$
\begin{aligned}
&\left\langle y^{m+1}, u\right\rangle+\beta \gamma\left\langle y_{z z z z}^{m+1}, u\right\rangle \\
&=- d_{m}\left\langle y^{-1}, u\right\rangle+\left(2 d_{m}-d_{m-1}\right)\left\langle y^{0}, u\right\rangle \\
&+\sum_{k=1}^{m-1}\left(-d_{k-1}+2 d_{k}-d_{k+1}\right)\left\langle y^{m-k}, u\right\rangle+\left(2 d_{0}-d_{1}\right)\left\langle y^{m}, u\right\rangle+\beta\left\langle f^{m+1}, u\right\rangle
\end{aligned}
$$

and

$$
\left\langle y^{1}, u\right\rangle+\beta \gamma\left\langle y_{z z z z}^{1}, u\right\rangle=-d_{0}\left\langle y^{-1}, u\right\rangle+2 d_{0}\left\langle y^{0}, u\right\rangle+\beta\left\langle f^{1}, u\right\rangle
$$

Definition 1 Let $g_{m}$ and $h_{m}, m=1,2, \ldots, N$, be the sequences which satisfy the inequality

$$
g_{m} \leq\left(\sum_{i=1}^{m-1} g_{i} h_{i}+\kappa\right), \quad m=1,2, \ldots, N
$$


where $g_{m} \geq 0, \kappa \geq 0$, then the following discrete Gronwall inequality holds:

$$
g_{m} \leq \kappa \cdot \exp \left(\sum_{i=1}^{m-1} g_{i}\right), \quad m=1,2, \ldots, N .
$$

\section{Stability and convergence}

The approach used in this section follows the general approach used in [19]. The stability and convergence analysis for semi-discrete problem are described in the following Theorems 1 and 2, respectively.

Theorem $1 \forall \Delta t>0$, the semi-discrete problem is unconditionally stable if

$$
\left\|y^{m+1}\right\|_{2} \leq c\left(\|\phi\|_{0}+\Delta t\|\psi\|_{0}+\beta\left\|f^{k+1}\right\|_{0}\right), \quad 0 \leq m \leq K-1 .
$$

Proof We apply mathematical induction to prove this theorem.

For $m=0$ and $u=y^{1}$, Eq. (30) can be expressed as

$$
\left\langle y^{1}, y^{1}\right\rangle+\beta \gamma\left\langle y_{x x x x}^{1}, y^{1}\right\rangle=-d_{0}\left\langle y^{-1}, y^{1}\right\rangle+2\left\langle y^{0}, y^{1}\right\rangle+\beta\left\langle f^{1}, y^{1}\right\rangle
$$

or

$$
\left\langle y^{1}, y^{1}\right\rangle+\beta \gamma\left\langle y_{z z}^{1}, y_{z z}^{1}\right\rangle=-d_{0}\left\langle y^{-1}, y^{1}\right\rangle+2 d_{0}\left\langle y^{0}, y^{1}\right\rangle+\beta\left\langle f^{1}, y^{1}\right\rangle .
$$

Here, all the boundary related contributions vanish because of the boundary constraints on $u$. Using $\|u\|_{0} \leq\|u\|_{2}$ and Schwarz's inequality, Eq. (34) becomes

$$
\begin{aligned}
\left\|y^{1}\right\|_{2}^{2} & \leq\left\|y^{-1}\right\|_{0}\left\|y^{1}\right\|_{0}+2\left\|y^{0}\right\|_{0}\left\|y^{1}\right\|_{0}+\beta\left\|f^{1}\right\|_{0}\left\|y^{1}\right\|_{0} \\
& \leq\left\|y^{-1}\right\|_{0}\left\|y^{1}\right\|_{2}+2\left\|y^{0}\right\|_{0}\left\|y^{1}\right\|_{2}+\beta\left\|f^{1}\right\|_{0}\left\|y^{1}\right\|_{2} \\
\left\|y^{1}\right\|_{2} & \leq\left\|y^{-1}\right\|_{0}+2\left\|y^{0}\right\|_{0}+\beta\left\|f^{1}\right\|_{0} \\
\left\|y^{1}\right\|_{2} & \leq\left(\|\phi\|_{0}-\Delta t\|\psi\|_{0}\right)+2\left\|\phi_{0}\right\|_{0}+\beta\left\|f^{1}\right\|_{0} \\
\left\|y^{1}\right\|_{2} & \leq 3\left(\|\phi\|_{0}-\Delta t\|\psi\|_{0}+\beta\left\|f^{1}\right\|_{0}\right)
\end{aligned}
$$

Hence

$$
\left\|y^{1}\right\|_{2} \leq c\left(\|\phi\|_{0}-\Delta t\|\psi\|_{0}+\beta\left\|f^{1}\right\|_{0}\right) .
$$

We assume that the result is true for $u=y^{k}$, i.e.,

$$
\left\|y^{k}\right\|_{2} \leq c\left(\left\|\phi_{0}\right\|_{0}+\Delta t\left\|\psi_{0}\right\|_{0}+\beta\left\|f^{k}\right\|_{0}\right), \quad k=2,3, \ldots, m .
$$

Let $u=y^{m+1}$ in Eq. (30)

$$
\begin{aligned}
\left\langle y^{m+1}, y^{m+1}\right\rangle+\beta \gamma\left\langle y_{z z z z}^{m+1}, y^{m+1}\right\rangle & \\
= & -d_{m}\left\langle y^{-1}, y^{m+1}\right\rangle+\left(2 d_{m}-d_{m-1}\right)\left\langle y^{0}, y^{m+1}\right\rangle \\
& +\sum_{k=1}^{m-1}\left(-d_{k-1}+2 d_{k}-d_{k+1}\right)\left\langle y^{m-k}, y^{m+1}\right\rangle+\left(2 d_{0}-d_{1}\right)\left\langle y^{m}, y^{m+1}\right\rangle+\beta\left\langle f^{m+1}, y^{m+1}\right\rangle .
\end{aligned}
$$


Integrating by parts gives

$$
\begin{aligned}
\left\langle y^{m+1}, y^{m+1}\right\rangle+\beta \gamma\left\langle y_{z z}^{m+1}, y_{z z}^{m+1}\right\rangle & \\
= & -d_{m}\left\langle y^{-1}, y^{m+1}\right\rangle+\left(2 d_{m}-d_{m-1}\right)\left\langle y^{0}, y^{m+1}\right\rangle \\
& +\sum_{k=1}^{m-1}\left(-d_{k-1}+2 d_{k}-d_{k+1}\right)\left\langle y^{m-k}, y^{m+1}\right\rangle+\left(2 d_{0}-d_{1}\right)\left\langle y^{m}, y^{m+1}\right\rangle+\beta\left\langle f^{m+1}, y^{m+1}\right\rangle .
\end{aligned}
$$

Using $\|u\|_{0} \leq\|u\|_{2}$ and Schwarz's inequality, the above expression then takes the following form:

$$
\begin{aligned}
\left\|y^{m+1}\right\|_{2}^{2} \leq & d_{m}\left\|y^{-1}\right\|_{0}\left\|y^{m+1}\right\|_{0}+\left(2 d_{m}-d_{m-1}\right)\left\|y^{0}\right\|_{0}\left\|y^{m+1}\right\|_{0}+\sum_{k=1}^{m-1}\left(-d_{k-1}+2 d_{k}\right. \\
& \left.\quad-d_{k+1}\right)\left\|y^{m-k}\right\|_{0}\left\|y^{m+1}\right\|_{0}+\left(2 d_{0}-d_{1}\right)\left\|y^{m}\right\|_{0}\left\|y^{m+1}\right\|_{0}+\beta\left\|f^{k+1}\right\|_{0}\left\|y^{m+1}\right\|_{0},
\end{aligned}
$$

or

$$
\begin{aligned}
\left\|y^{m+1}\right\|_{2}^{2} \leq & d_{m}\left\|y^{-1}\right\|_{0}\left\|y^{m+1}\right\|_{2}+\left(2 d_{m}-d_{m-1}\right)\left\|y^{0}\right\|_{0}\left\|y^{m+1}\right\|_{2}+\sum_{k=1}^{m-1}\left(-d_{k-1}+2 d_{k}\right. \\
& \left.-d_{k+1}\right)\left\|y^{m-k}\right\|_{0}\left\|y^{m+1}\right\|_{2}+\left(2 d_{0}-d_{1}\right)\left\|y^{m}\right\|_{0}\left\|y^{m+1}\right\|_{2}+\beta\left\|f^{k+1}\right\|_{0}\left\|y^{m+1}\right\|_{2} \\
\left\|y^{m+1}\right\|^{2} \leq & d_{m}\left\|y^{-1}\right\|_{0}+\left(2 d_{m}-d_{m-1}\right)\left\|y^{0}\right\|_{0}+\sum_{k=1}^{m-1}\left(-d_{k-1}+2 d_{k}-d_{k+1}\right)\left\|y^{m-k}\right\|_{0} \\
& +\left(2 d_{0}-d_{1}\right)\left\|y^{m}\right\|_{0}+\beta\left\|f^{k+1}\right\|_{0} .
\end{aligned}
$$

Using (32), the above relation can be written as follows:

$$
\begin{aligned}
\left\|y^{m+1}\right\|_{2} \leq & \left(\left(2 d_{m}-d_{m-1}\right)\left\|y^{0}\right\|_{0}+d_{m}\left\|y^{-1}\right\|_{0}+\beta\left\|f^{m+1}\right\|_{0}\right) \exp \left[\left(2 d_{0}-d_{1}\right)\right. \\
& \left.+\sum_{k=1}^{m-1}\left(-d_{k-1}+2 d_{k}-d_{k+1}\right)\right] \\
\left\|y^{m+1}\right\|_{2} \leq & \left(\left\|y^{0}\right\|_{0}+\left\|y^{-1}\right\|_{0}+\beta\left\|f^{k+1}\right\|_{0}\right) \exp \left(1+d_{m-1}-d_{m}\right), \\
\left\|y^{m+1}\right\|_{2} \leq & \left(\|\phi\|_{0}+\|\phi\|_{0}-\Delta t\|\psi\|_{0}+\beta\left\|f^{k+1}\right\|_{0}\right) \exp \left(1+d_{m-1}-d_{m}\right), \\
\left\|y^{m+1}\right\|_{2} \leq & c\left(\|\phi\|_{0}-\Delta t\|\psi\|_{0}+\beta\left\|f^{k+1}\right\|_{0}\right) .
\end{aligned}
$$

Theorem 2 The numerical solution obtained by the proposed method converges to the exact solution if the following relation holds:

$$
\left\|y\left(t_{m}\right)-y^{m}\right\|_{2} \leq \zeta \Delta t^{2}, \quad m=1,2, \ldots, K
$$

where $\zeta$ is constant and $\zeta=\left(1+d_{m-1}\right) \exp \left(1+d_{m-1}-d_{m}\right)$.

Proof Consider $e^{m}=y\left(z, t_{m}\right)-y^{m}(z)$ for $m=1$, using Eqs. (1) and (30), we have

$$
\left\langle e^{1}, u\right\rangle+\beta \gamma\left\langle e_{z z}^{1}, u_{z z}\right\rangle=\left\langle e^{-1}, u\right\rangle+2 d_{0}\left\langle e^{0}, u\right\rangle+\left\langle l^{1}, u\right\rangle, \quad \forall u \in H_{0}^{2}(\eta) .
$$


Again using $\|u\|_{0} \leq\|u\|_{2}$, Schwarz's inequality, $u=e^{1}$, and $e^{0}=0$, we get

$$
\begin{aligned}
& \left\|e^{1}\right\|_{2}^{2} \leq\left\|e^{-1}\right\|_{0}+\left\|e^{1}\right\|_{0}+\left\|l^{1}\right\|_{0}\left\|e^{1}\right\|_{0^{\prime}} \\
& \left\|e^{1}\right\|_{2}^{2} \leq\left\|e^{-1}\right\|_{0}+\left\|e^{1}\right\|_{2}+\left\|l^{1}\right\|_{0}\left\|e^{1}\right\|_{2}, \\
& \left\|e^{1}\right\|_{2} \leq\left\|e^{-1}\right\|_{0}+\left\|l^{1}\right\|_{0} .
\end{aligned}
$$

Since $\left\|e^{-1}\right\| \leq \Delta t^{2}$, using Eq. (27) leads to

$$
\begin{aligned}
& \left\|y\left(t_{1}\right)-y^{1}\right\|_{2} \leq 2 \Delta t^{2}, \\
& \left\|y\left(t_{1}\right)-y^{1}\right\|_{2} \leq \zeta \Delta t^{2} .
\end{aligned}
$$

For $m=1$, Eq. (38) is true.

Now, consider (38) is satisfied for $m=(1) r$, i.e.,

$$
\left\|y\left(t_{m}\right)-y^{m}\right\|_{2} \leq \zeta \Delta t^{2}
$$

Using (1), (29), (30) and for $m=r+1$, the error equation is derived as follows:

$$
\begin{aligned}
\left\langle e^{m+1}, u\right\rangle+\beta \gamma\left\langle e_{z z}^{m+1}, u_{z z}\right\rangle & \\
= & -d_{m}\left\langle e^{-1}, u\right\rangle+\left(2 d_{m}-d_{m-1}\right)\left\langle e^{0}, u\right\rangle \\
& +\sum_{k=1}^{m-1}\left(-d_{k-1}+2 d_{k}-d_{k+1}\right)\left\langle e^{m-k}, u\right\rangle+\left(2 d_{0}-d_{1}\right)\left\langle e^{m}, u\right\rangle+\left\langle l^{m+1}, u\right\rangle .
\end{aligned}
$$

Now, using the induction assumption and taking $u=e^{m+1}$, Eq. (40) can be written as follows:

$$
\begin{aligned}
\left\|e^{m+1}\right\|_{2}^{2} \leq & -d_{m}\left\|e^{-1}\right\|_{0}\left\|e^{m+1}\right\|_{0}+\left(2 d_{m}-d_{m-1}\right)+\left\|e^{0}\right\|_{0}\left\|e^{m+1}\right\|_{0} \\
& +\sum_{k=1}^{m-1}\left(-d_{k-1}+2 d_{k}-d_{k+1}\right)\left\|e^{m-k}\right\|_{0}\left\|e^{m+1}\right\|_{0}+\left(2 d_{0}-d_{1}\right)\left\|e^{m}\right\|_{0}\left\|e^{m+1}\right\|_{0} \\
& +\left\|l^{m+1}\right\|_{0}\left\|e^{m+1}\right\|_{0}, \\
\left\|e^{m+1}\right\|_{2} \leq & -d_{m}\left\|e^{-1}\right\|_{0}+\sum_{k=1}^{m-1}\left(-d_{k-1}+2 d_{k}-d_{k+1}\right)\left\|e^{m-k}\right\|_{2}+\left(2 d_{0}-d_{1}\right)\left\|e^{m}\right\|_{2}+\left\|l^{m+1}\right\|_{0} .
\end{aligned}
$$

Using (34), we have

$$
\left\|e^{m+1}\right\|_{2} \leq\left(d_{m}\left\|e^{-1}\right\|_{0}+\left\|l^{m+1}\right\|_{0}\right) \exp \left[\sum_{k=1}^{m-1}\left(-d_{k-1}+2 d_{k}-d_{k+1}\right)+2 d_{0}-d_{1}\right],
$$

or

$$
\begin{aligned}
\left\|e^{m+1}\right\|_{2} & \leq\left(d_{m}\left\|e^{-1}\right\|_{0}+\left\|l^{m+1}\right\|_{0}\right) \exp \left(1+d_{k-1}-d_{k}\right), \\
\left\|e^{m+1}\right\|_{2} & \leq\left(d_{m} \Delta t^{2}+\Delta t^{2}\right)+\exp \left(1+d_{k-1}-d_{k}\right), \\
\left\|e^{m+1}\right\|_{2} & \leq \zeta \Delta t^{2} .
\end{aligned}
$$

Hence, proved. 


\section{Spatial discretization}

The approach used in this section follows the general approach used in [19].

Let $\left(z_{i}, t_{m}\right)$ be the grid points of a uniform mesh to discretize the region $[0, L] \times[0, T]$, where $z_{i}=i h, i=0,1,2, \ldots, N$, and $h=\frac{L}{N}$. The spatial discretization of Eq. (21) using quintic non-polynomial spline is formulated as follows:

$$
\begin{aligned}
Y_{i}^{m+1}(z)+\beta \gamma F^{m+1} \\
=-d_{m} Y^{-1}(z)+\left(2 d_{m}-d_{m-1}\right) Y^{0}(z)+\sum_{k=1}^{m-1}\left(-d_{k-1}+2 d_{k}-d_{k+1}\right) Y^{m-k}(z) \\
\quad+\left(2 d_{0}-d_{1}\right) Y^{m}(z)+\beta f^{m+1}(z), \quad m=1,2,3, \ldots, K-1 .
\end{aligned}
$$

The operator $\varphi$ is defined as

$$
\varphi Y_{k}=\mu_{1} Y_{k-2}+v_{1} Y_{k-1}+\kappa_{1} Y_{k}+v_{1} Y_{k+1}+\mu_{1} Y_{k+2}
$$

Now, Eq. (7) takes the following form:

$$
\varphi F_{i}=\frac{1}{h^{4}}\left(Y_{i-2}-4 Y_{i-1}+6 Y_{i}-4 Y_{i+1}+Y_{i+2}\right) .
$$

Applying $\varphi$ on Eq. (41), we obtain

$$
\begin{aligned}
\left(\mu_{1} Y_{i-2}^{m+1}+v_{1} Y_{i-1}^{m+1}+\kappa_{1} Y_{i}^{m+1}+v_{1} Y_{i+1}^{m+1}+\mu_{1} Y_{i+2}^{m+1}\right)+\frac{\beta \gamma}{h^{4}}\left(Y_{i-2}^{m+1}-4 Y_{i-1}^{m+1}+6 Y_{i}^{m+1}\right. \\
\left.\quad-4 Y_{i+1}^{m+1}+Y_{i+2}^{m+1}\right) \\
=-d_{m}\left(\mu_{1} Y_{i-2}^{-1}+v_{1} Y_{i-1}^{-1}+\kappa_{1} Y_{i}^{-1}+v_{1} Y_{i+1}^{-1}+\mu_{1} Y_{i+2}^{-1}\right)+\left(2 d_{m}\right. \\
\left.\quad-d_{m-1}\right)\left(\mu_{1} Y_{i-2}^{0}+v_{1} Y_{i-1}^{0}+\kappa_{1} Y_{i}^{0}+v_{1} Y_{i+1}^{0}+\mu_{1} Y_{i+2}^{0}\right) \\
\quad+\sum_{k=1}^{m-1}\left(-d_{k-1}+2 d_{k}-d_{k+1}\right)\left(\mu_{1} Y_{i-2}^{m-k}\right. \\
\left.\quad+v_{1} Y_{i-1}^{m-k}+\kappa_{1} Y_{i}^{m-k}+v_{1} Y_{i+1}^{m-k}+\mu_{1} Y_{i+2}^{m-k}\right) \\
\quad+\left(2 d_{0}-d_{1}\right)\left(\mu_{1} Y_{i-2}^{m}+v_{1} Y_{i-1}^{m}+\kappa_{1} Y_{i}^{m}+v_{1} Y_{i+1}^{m}\right. \\
\left.\quad+\mu_{1} Y_{i+2}^{m}\right)+\beta\left(\mu_{1} f_{i-2}^{m+1}+v_{1} f_{i-1}^{m+1}+\kappa_{1} f_{i}^{m+1}+v_{1} f_{i+1}^{m+1}+\mu_{1} f_{i+2}^{m+1}\right) \\
1 \leq m \leq K-1 .
\end{aligned}
$$

After simplification, (44) takes the following form:

$$
\begin{aligned}
\left(\mu_{1}\right. & \left.+\frac{\beta \gamma}{h^{4}}\right) Y_{i-2}^{m+1}+\left(v_{1}-4 \frac{\beta \gamma}{h^{4}}\right) Y_{i-1}^{m+1}+\left(\kappa_{1}+6 \frac{\beta \gamma}{h^{4}}\right) Y_{i}^{m+1} \\
& +\left(v_{1}-4 \frac{\beta \gamma}{h^{4}}\right) Y_{i+1}^{m+1}+\left(\mu_{1}+\frac{\beta \gamma}{h^{4}}\right) Y_{i+2}^{m+1} \\
= & Q_{i}, \quad 2 \leq i \leq N-2,1 \leq m \leq K-1,
\end{aligned}
$$


where

$$
\begin{aligned}
Q_{i}= & -d_{m}\left(\mu_{1} Y_{i-2}^{-1}+v_{1} Y_{i-1}^{-1}+\kappa_{1} Y_{i}^{-1}+v_{1} Y_{i+1}^{-1}+\mu_{1} Y_{i+2}^{-1}\right)+\left(2 d_{m}-d_{m-1}\right)\left(\mu_{1} Y_{i-2}^{0}\right. \\
& \left.+v_{1} Y_{i-1}^{0}+\kappa_{1} Y_{i}^{0}+v_{1} Y_{i+1}^{0}+\mu_{1} Y_{i+2}^{0}\right)+\sum_{k=1}^{m-1}\left(-d_{k-1}+2 d_{k}-d_{k+1}\right)\left(\mu_{1} Y_{i-2}^{m-k}+v_{1} Y_{i-1}^{m-k}\right. \\
& \left.+\kappa_{1} Y_{i}^{m-k}+v_{1} Y_{i+1}^{m-k}+\mu_{1} Y_{i+2}^{m-k}\right)+\left(2 d_{0}-d_{1}\right)\left(\mu_{1} Y_{i-2}^{m}+v_{1} Y_{i-1}^{m}+\kappa_{1} Y_{i}^{m}+v_{1} Y_{i+1}^{m}\right. \\
& \left.+\mu_{1} Y_{i+2}^{m}\right)+\beta\left(\mu_{1} f_{i-2}^{m+1}+v_{1} f_{i-1}^{m+1}+\kappa_{1} f_{i}^{m+1}+v_{1} f_{i+1}^{m+1}+\mu_{1} f_{i+2}^{m+1}\right), \quad 1 \leq m \leq K-1 .
\end{aligned}
$$

The above system yields $(N-3)$ equations involving $(N-1)$ unknowns $Y_{i}^{m+1}, 1 \leq i \leq N-1$. We extract two more equations from boundary conditions as follows:

$$
\begin{aligned}
\left(\varpi_{0}\right. & \left.-2 \frac{\beta \gamma}{h^{4}}\right) Y_{0}^{m+1}+\left(\varpi_{1}+5 \frac{\beta \gamma}{h^{4}}\right) Y_{1}^{m+1}+\left(\varpi_{2}-4 \frac{\beta \gamma}{h^{4}}\right) Y_{2}^{m+1}+\left(\varpi_{3}+\frac{\beta \gamma}{h^{4}}\right) Y_{3}^{m+1} \\
= & \left(2 d_{0}-d_{1}\right)\left(\varpi_{0} Y_{0}^{m}+\varpi_{1} Y_{1}^{m}+\varpi_{2} Y_{2}^{m}+\varpi_{3} Y_{3}^{m}\right) \\
& +\sum_{k=1}^{m-1}\left(d_{k}-d_{k+1}\right)\left(\varpi_{0} Y_{0}^{m-k}+\varpi_{1} Y_{1}^{m-k}+\varpi_{2} Y_{2}^{m-k}+\varpi_{3} Y_{3}^{m-k}\right) \\
& +\left(d_{m}-d_{m-1}\right)\left(\varpi_{0} \phi_{0}+\varpi_{1} \phi_{1}+\varpi_{2} \phi_{2}+\varpi_{3} \phi_{3}\right) \\
& +\Delta d_{m}\left(\varpi_{0} \psi_{0}+\varpi_{1} \psi_{1}+\varpi_{2} \psi_{2}+\varpi_{3} \psi_{3}\right) \\
& +\beta\left(\varpi_{0} f_{0}^{m+1}+\varpi_{1} f_{1}^{m+1}+\varpi_{2} f_{2}^{m+1}+\varpi_{3} f_{3}^{m+1}\right) .
\end{aligned}
$$

Similarly,

$$
\begin{aligned}
\left(\varpi_{3}\right. & \left.+\frac{\beta \gamma}{h^{4}}\right) Y_{N-3}^{m+1}+\left(\varpi_{2}-4 \frac{\beta \gamma}{h^{4}}\right) Y_{N-2}^{m+1}+\left(\varpi_{1} 5 \frac{\beta \gamma}{h^{4}}\right) Y_{N-1}^{m+1}+\left(\varpi_{0}-2 \frac{\beta \gamma}{h^{4}}\right) Y_{N}^{m+1} \\
= & \left(2 d_{0}-d_{1}\right)\left(\varpi_{3} Y_{N-3}^{m}+\varpi_{2} Y_{N-2}^{m}+\varpi_{1} Y_{N-1}^{m}+\varpi_{0} Y_{N}^{m}\right) \\
& +\sum_{k=1}^{m-1}\left(d_{k}-d_{k+1}\right)\left(\varpi_{3} Y_{N-3}^{m-k}+\varpi_{2} Y_{N-2}^{m-k}+\varpi_{1} Y_{N-1}^{m-k}+\varpi_{0} Y_{N}^{m-k}\right) \\
& +\left(d_{m}-d_{m-1}\right)\left(\varpi_{3} \phi_{N-3}+\varpi_{2} \phi_{N-2}+\varpi_{1} \phi_{N-1}+\varpi_{0} \phi_{N}\right) \\
& +\Delta d_{m}\left(\varpi_{3} \psi_{N-3}+\varpi_{2} \psi_{N-2}+\varpi_{1} \psi_{N-1}\right. \\
& \left.+\varpi_{0} \psi_{N}\right)+\beta\left(\varpi_{3} f_{N-3}^{m+1}+\varpi_{2} f_{N-2}^{m+1}+\varpi_{1} f_{N-1}^{m+1}+\varpi_{0} f_{N}^{m+1}\right) .
\end{aligned}
$$

In order to apply this scheme, $Y^{2}=\left[Y_{1}^{2}, Y_{2}^{2}, Y_{3}^{2}, \ldots, Y_{N-1}^{2}\right]^{T}$ and $Y^{1}=\left[Y_{1}^{1}, Y_{2}^{1}, Y_{3}^{1}, \ldots\right.$, $\left.Y_{N-1}^{1}\right]^{T}$ are required. Solving (25) and utilizing the quintic non-polynomial spline, $Y^{1}$ is calculated as follows:

$$
\begin{aligned}
& \left(\mu_{1}+\frac{\beta \gamma}{h^{4}}\right) Y_{i-2}^{1}+\left(v_{1}-4 \frac{\beta \gamma}{h^{4}}\right) Y_{i-1}^{1}+\left(\kappa_{1}+6 \frac{\beta \gamma}{h^{4}}\right) Y_{i}^{1}+\left(v_{1}-4 \frac{\beta \gamma}{h^{4}}\right) Y_{i+1}^{1} \\
& +\left(\mu_{1}+\frac{\beta \gamma}{h^{4}}\right) Y_{i+2}^{1}=J_{i}, \quad 2 \leq i \leq N-2,
\end{aligned}
$$


where

$$
\begin{aligned}
J_{i}= & \left(\mu_{1} \phi_{i-2}+v_{1} \phi_{i-1}+\kappa_{1} \phi_{i}+v_{1} \phi_{i+1}+\mu_{1} \phi_{i+2}\right)+\Delta t\left(\mu_{1} \psi_{i-2}^{1}+v_{1} \psi_{i-1}^{1}+\kappa_{1} \psi_{i}^{1}\right. \\
& \left.+v_{1} \psi_{i+1}^{1}+\mu_{1} \psi_{i+2}^{1}\right)+\beta\left(\mu_{1} f_{i-2}^{1}+v_{1} f_{i-1}^{1}+\kappa_{1} f_{i}^{1}+v_{1} f_{i+1}^{1}+\mu_{1} f_{i+2}^{1}\right) .
\end{aligned}
$$

System (48) contains $(N-1)$ unknowns $Y_{i}^{1}, 1 \leq i \leq N-1$, involved in $(N-3)$ equations. We extract two more equations from the end conditions as follows:

$$
\begin{aligned}
\left(\varpi_{0}\right. & \left.-2 \frac{\beta \gamma}{h^{4}}\right) Y_{0}^{1}+\left(\varpi_{1}+5 \frac{\beta \gamma}{h^{4}}\right) Y_{1}^{1}+\left(\varpi_{2}-4 \frac{\beta \gamma}{h^{4}}\right) Y_{2}^{1}+\left(\varpi_{3}+\frac{\beta \gamma}{h^{4}}\right) Y_{3}^{1} \\
= & \left(\varpi_{0} \phi_{0}+\varpi_{1} \phi_{1}+\varpi_{2} \phi_{2}+\varpi_{3} \phi_{3}\right)+\Delta t\left(\varpi_{0} \psi_{0}+\varpi_{1} \psi_{1}+\varpi_{2} \psi_{2}+\varpi_{3} \psi_{3}\right) \\
& +\beta\left(\varpi_{0} f_{0}^{1}+\varpi_{1} f_{1}^{1}+\varpi_{2} f_{2}^{1}+\varpi_{3} f_{3}^{1}\right)
\end{aligned}
$$

and

$$
\begin{aligned}
\left(\varpi_{3}\right. & \left.+\frac{\beta \gamma}{h^{4}}\right) Y_{N-3}^{1}+\left(\varpi_{2}-4 \frac{\beta \gamma}{h^{4}}\right) Y_{N-2}^{1}+\left(\varpi_{1}+5 \frac{\beta \gamma}{h^{4}}\right) Y_{N-1}^{1}+\left(\varpi_{0}-2 \frac{\beta \gamma}{h^{4}}\right) Y_{N}^{1} \\
= & \left(\varpi_{3} \phi_{N-3}+\varpi_{2} \phi_{N-2}+\varpi_{1} \phi_{N-1}+\varpi_{0} \phi_{N}\right)+\Delta t\left(\varpi_{3} \psi_{N-3}+\varpi_{2} \psi_{N-2}\right. \\
& \left.+\varpi_{1} \psi_{N-1}+\varpi_{0} \psi_{N}\right)+\beta\left(\varpi_{3} f_{N-3}^{1}+\varpi_{2} f_{N-2}^{1}+\varpi_{1} f_{N-1}^{1}+\varpi_{0} f_{N}^{1}\right) .
\end{aligned}
$$

Suppose $\phi=\left[\phi_{1}, \phi_{2}, \ldots, \phi_{N-1}\right]^{T}, f=\left[f_{1}, f_{2}, \ldots, f_{N-1}\right]^{T}, \widetilde{\phi}=\left[\phi_{0}, 0, \ldots, 0, \phi_{N}\right]^{T}$, and $\tilde{f}=\left[f_{0}, 0\right.$, $\left.\ldots, 0, f_{N}\right]^{T}$ are column matrices with $(N-1)$ entries. The system in $(48)-(50)$ can be expressed as

$$
A Y^{1}=B\left(\phi+\Delta t \psi+\beta f^{1}\right)+C(\tilde{\phi}+\Delta t \tilde{\psi}+\beta \tilde{f})-D
$$

Here, $A, B$, and $C$ are square matrices of order $n-1$.

$$
\begin{aligned}
& A=\left(\begin{array}{cccccccc}
\varpi_{1}+5 \frac{\beta \gamma}{h^{4}} & \varpi_{2}-4 \frac{\beta \gamma}{h^{4}} & \varpi_{3}+\frac{\beta \gamma}{h^{4}} & 0 & 0 & 0 & \ldots & 0 \\
v_{1}-4 \frac{\beta_{\gamma}}{h^{4}} & \kappa_{1}+6 \frac{\beta \gamma}{h^{4}} & \nu_{1}-4 \frac{\beta \gamma}{h^{4}} & \mu_{1}+\frac{\beta \gamma}{h^{4}} & 0 & 0 & \ldots & 0 \\
\mu_{1}+\frac{\beta \gamma}{h^{4}} & \nu_{1}-4 \frac{\beta \gamma}{h^{4}} & \kappa_{1}+6 \frac{\beta \gamma}{h^{4}} & \nu_{1}-4 \frac{\beta \gamma}{h^{4}} & \mu_{1}+\frac{\beta \gamma}{h^{4}} & 0 & \ldots & 0 \\
& \ddots & & & \ddots & & \ddots & \\
0 & \ldots & 0 & \mu_{1}+\frac{\beta \gamma}{h^{4}} & \nu_{1}-4 \frac{\beta \gamma}{h^{4}} & \kappa_{1}+6 \frac{\beta \gamma}{h^{4}} & 0 v_{1}-4 \frac{\beta \gamma}{h^{4}} & \mu_{1}+\frac{\beta \gamma}{h^{4}} \\
0 & \ldots & 0 & & \mu_{1}+\frac{\beta \gamma}{h^{4}} & \nu_{1}-4 \frac{\beta \gamma \gamma}{h^{4}} & \kappa_{1}+6 \frac{\beta \gamma}{h^{4}} & \nu_{1}-4 \frac{\beta \gamma}{h^{4}} \\
0 & \cdots & 0 & 0 & 0 & \omega_{3}+\frac{\beta \gamma}{h^{4}} & \varpi_{2}-4 \frac{\beta \gamma}{h^{4}} & \varpi_{1}+5 \frac{\beta \gamma}{h^{4}}
\end{array}\right) \text {, } \\
& B=\left(\begin{array}{cccccccc}
\varpi_{1} & \varpi_{2} & \varpi_{3} & 0 & 0 & 0 & \cdots & 0 \\
v_{1} & \kappa_{1} & v_{1} & \mu_{1} & 0 & 0 & \cdots & 0 \\
\mu_{1} & v_{1} & \kappa_{1} & v_{1} & \mu_{1} & 0 & \cdots & 0 \\
& \ddots & & & \ddots & & \ddots & \\
0 & \cdots & 0 & \mu_{1} & v_{1} & \kappa_{1} & \nu_{1} & \mu_{1} \\
0 & \cdots & 0 & 0 & \mu_{1} & v_{1} & \kappa_{1} & v_{1} \\
0 & \cdots & 0 & 0 & 0 & \varpi_{3} & \varpi_{2} & \varpi_{1}
\end{array}\right) \text { and }
\end{aligned}
$$




$$
\begin{aligned}
C & =\left(\begin{array}{ccccccccc}
\varpi_{0} & 0 & 0 & 0 & 0 & 0 & 0 & \cdots & 0 \\
1 & 0 & 0 & 0 & 0 & 0 & 0 & \cdots & 0 \\
0 & 0 & 0 & 0 & 0 & 0 & 0 & \cdots & 0 \\
& \ddots & & & \ddots & & \ddots & & \\
0 & \cdots & 0 & 0 & 0 & 0 & 0 & 0 & 0 \\
0 & \cdots & 0 & 0 & 0 & 0 & 0 & 0 & 1 \\
0 & \cdots & 0 & 0 & 0 & 0 & 0 & 0 & \varpi_{0}
\end{array}\right) . \\
D & =\left[-M_{0} h^{2}, 0,0,0, \ldots,-M_{N} h^{2}\right]^{T} .
\end{aligned}
$$

\section{Numerical results and discussion}

In this section, we present the simulation results for two test examples in order to validate the proposed numerical algorithm. All calculations are performed in Mathematica 10.0. The accuracy of the current scheme is verified by the error norms $L_{\infty}, L_{2}$ and experimental order of convergence (EOC) [22, 23]:

$$
L_{\infty}=\max \left|y_{j}-Y_{j}\right|, \quad L_{2}=\sqrt{\frac{\sum_{j=0}^{N}\left|y_{j}-Y_{j}\right|^{2}}{\sum_{j=0}^{N}\left|y_{j}\right|^{2}}}, \quad E O C=\frac{1}{\log (2)} \log \left[\frac{L_{\infty}(2 N)}{L_{\infty}(N)}\right],
$$

where $y_{j}, Y_{j}$ are the exact analytical and quintic non-polynomial spline solutions at $j$ th nodal point respectively.

Problem 1 Consider problem (1) with $\gamma=0.05$ [1].

The exact analytical solution of this problem is

$$
y(z, t)=2(t+1) \sin ^{2}(z) .
$$

The maximum and Euclidean error norms corresponding to four different values of $\Delta t$ are reported in Table 1 using $N=100$ and $\alpha=1.75$. It is clear that the presented scheme approximates the exact analytical solution more precisely as compared to the method used

Table 1 Error norms for Problem 1 at $t=1$ with $N=100, \alpha=1.75$

\begin{tabular}{llllll}
\hline$\Delta t$ & \multicolumn{2}{l}{ Method in [1] } & & & \multicolumn{2}{l}{ Proposed method } \\
\cline { 2 - 3 } & $L_{\infty}$ & & $L_{\infty}$ & $L_{2}$ & $2.3182 \times 10^{-7}$ \\
0.001 & $1.8221 \times 10^{-3}$ & $1.1556 \times 10^{-4}$ & & $1.5535 \times 10^{-6}$ & $6.2981 \times 10^{-8}$ \\
0.0005 & $5.6177 \times 10^{-4}$ & $2.9965 \times 10^{-5}$ & & $5.3380 \times 10^{-7}$ & $2.1050 \times 10^{-8}$ \\
0.00025 & $1.5380 \times 10^{-4}$ & $9.9200 \times 10^{-6}$ & & $1.9124 \times 10^{-7}$ & $6.4940 \times 10^{-9}$ \\
0.000125 & $3.9312 \times 10^{-5}$ & $1.9910 \times 10^{-6}$ & & $5.6215 \times 10^{-8}$ & \\
\hline
\end{tabular}

Table 2 Error norms for Problem 1, with $N=80$ and $\Delta t=0.001$

\begin{tabular}{lllll}
\hline$\alpha$ & & $t=0.25$ & $t=0.5$ & $t=1$ \\
\hline 1.25 & $L_{2}$ & $2.5869 \times 10^{-7}$ & $2.0680 \times 10^{-7}$ & $1.5465 \times 10^{-7}$ \\
& $L_{\infty}$ & $2.0113 \times 10^{-6}$ & $1.4491 \times 10^{-6}$ & $9.9176 \times 10^{-7}$ \\
1.50 & $L_{2}$ & $2.4923 \times 10^{-7}$ & $2.2555 \times 10^{-7}$ & $1.9223 \times 10^{-7}$ \\
& $L_{\infty}$ & $2.0700 \times 10^{-6}$ & $1.6562 \times 10^{-6}$ & $1.2714 \times 10^{-6}$ \\
1.75 & $L_{2}$ & $2.3392 \times 10^{-7}$ & $2.3887 \times 10^{-7}$ & $2.3182 \times 10^{-7}$ \\
& $L_{\infty}$ & $2.0669 \times 10^{-6}$ & $1.8185 \times 10^{-6}$ & $1.5535 \times 10^{-6}$ \\
\hline
\end{tabular}


Table 3 Experimental order of convergence for Problem 1, when $\alpha=1.50, N=80, \Delta t=0.001$

\begin{tabular}{lllll}
\hline$N$ & $L_{\infty}$ & $E O C$ & $L_{2}$ & $E O C$ \\
\hline 10 & $9.8950 \times 10^{-2}$ & - & $2.5950 \times 10^{-2}$ & - \\
20 & $6.0451 \times 10^{-3}$ & 4.0329 & $1.4778 \times 10^{-3}$ & 4.1342 \\
40 & $3.7842 \times 10^{-4}$ & 3.9977 & $8.5142 \times 10^{-5}$ & 4.1174 \\
80 & $2.1927 \times 10^{-5}$ & 4.1092 & $6.0168 \times 10^{-6}$ & 3.8228 \\
\hline
\end{tabular}

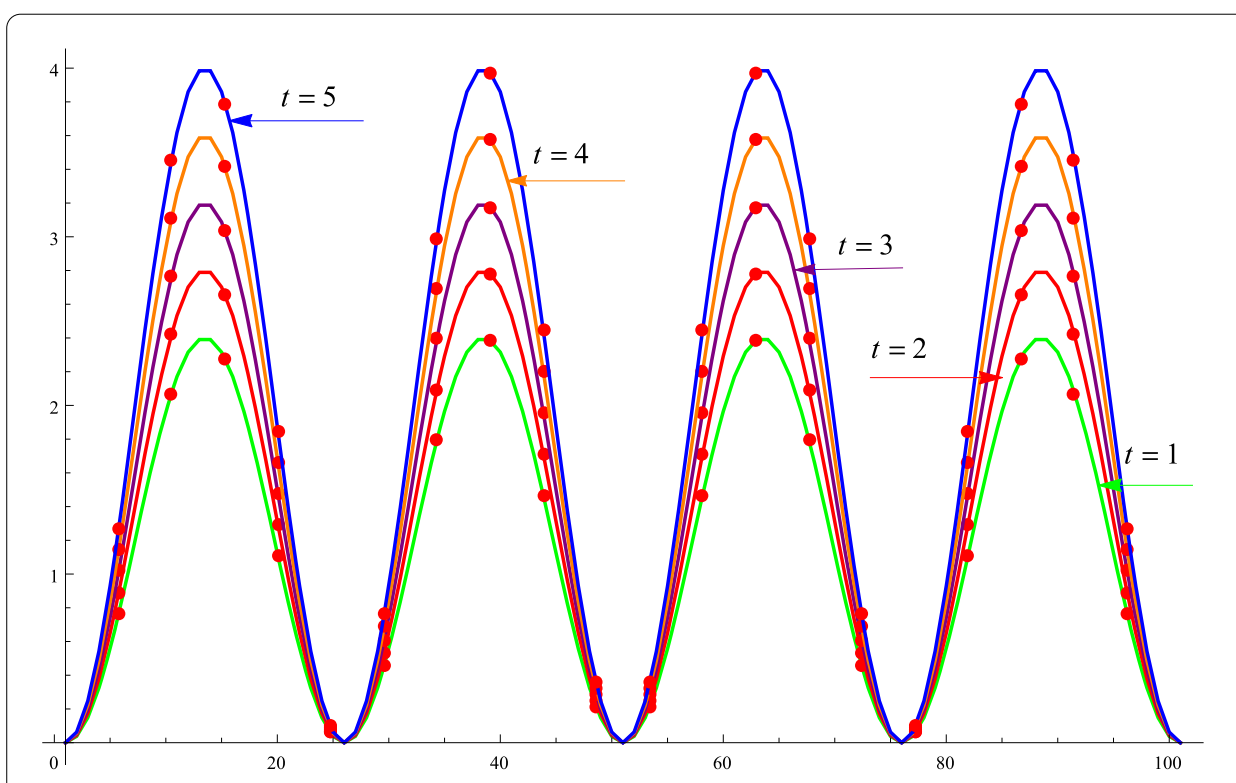

Figure 1 Approximate solution for Problem 1 at different time levels when $\Delta t=0.001, N=80, \alpha=1.75$

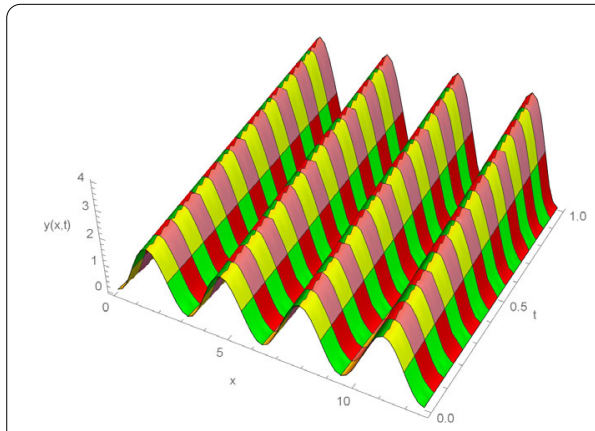

(a) Exact

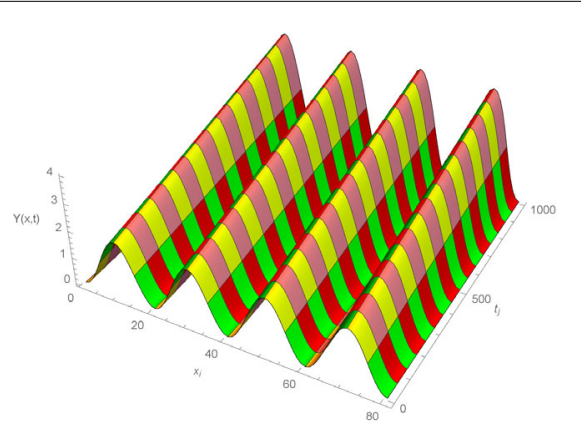

(b)Approximate

Figure 2 Exact and numerical solution for Problem 1 with $N=80, \Delta t=0.001$, and $\alpha=1.50$

in [1]. In Table 2, the error norms $L_{\infty}$ and $L_{2}$ are listed at $t=0.25,0.5,1$ using different values of $\alpha$. The calculations of slope rate of convergence in spatial direction are presented in Table 3 when error norms are calculated for $\Delta t=0.001, \alpha=1.50$. The computational rate of convergence of the proposed method in spatial direction is in line with theoretical results even with a larger time step. Figure 1 displays the physical behaviour of numerically approximated solution at various time stages. The three-dimensional visuals of exact analytical and non-polynomial quintic spline solutions are shown in Fig. 2 using $\alpha=1.50$, $N=100$, and $\Delta t=0.001$. From Fig. 2 , it is clear that the numerical solution is consistent 


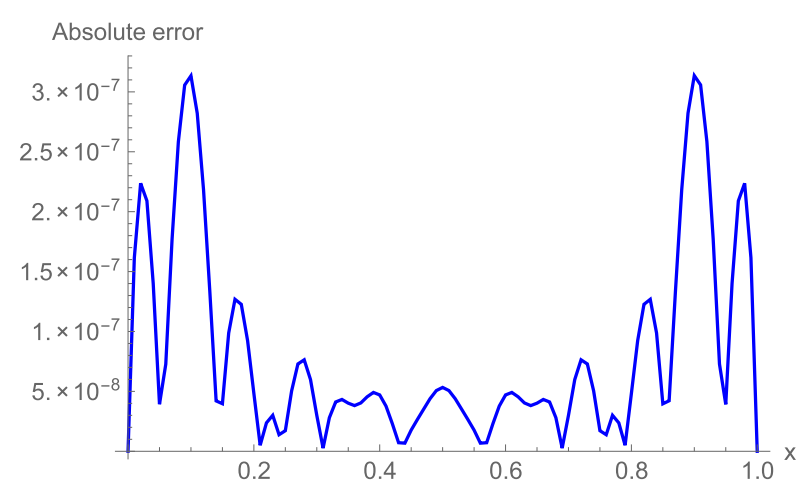

Figure 3 Absolute error for Problem 1 when $N=100, \alpha=1.75$, and $\Delta t=0.0005$

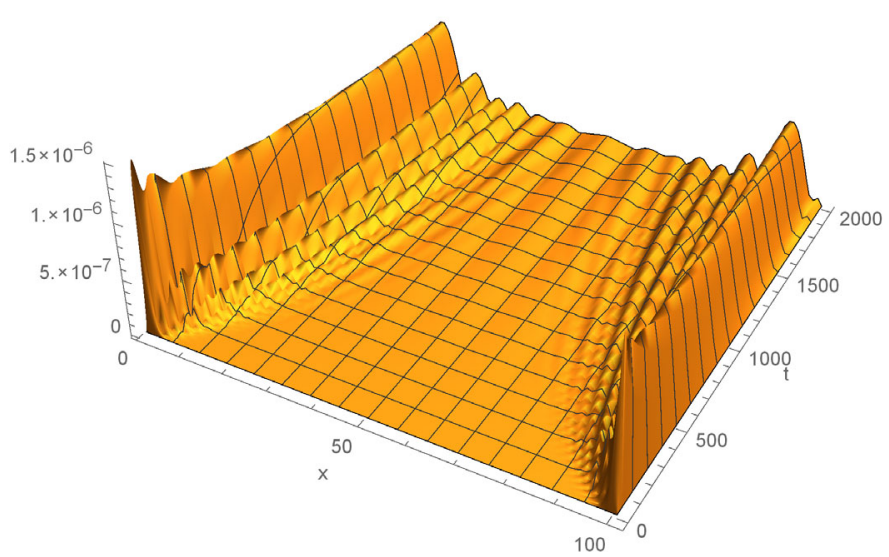

Figure 4 Absolute error for Problem 1 when $N=100, \alpha=1.5$ and $t=1$

with the exact solution, which indicates that the proposed method is effective. Figure 3 displays the absolute error at $t=1$ with $\alpha=1.75$ and $N=100$, whereas Fig. 4 represents 3D error plot using $N=100, \alpha=1.5$, and $\Delta t=0.001$.

Problem 2 As a second experiment, consider the following fourth order superdiffusion equation [1]:

$$
\frac{\partial^{\alpha} y}{\partial t^{\alpha}}+\gamma \frac{\partial^{4} y}{\partial z^{4}}=f(z, t), \quad z \in[0,1], t \in[0, T]
$$

the initial/end conditions are

$$
\begin{aligned}
& y(z, 0)=\frac{1}{\pi^{5}}\left(\pi^{10} \sin (\pi z)+\cos (\pi z)-\cos (3 \pi z)\right), \\
& y(0, t)=y(1, t)=0, \\
& y_{z z}(0, t)=\frac{1}{\pi^{3}} 8(1+t), \quad y_{z z}(1, t)=-\frac{1}{\pi^{3}} 8(1+t) .
\end{aligned}
$$


Table 4 Error norms for Problem 2 at $t=1$ with $N=80, \alpha=1.50$

\begin{tabular}{llllll}
\hline$\Delta t$ & \multicolumn{2}{l}{ Method in [1] } & & & \multicolumn{2}{l}{ Proposed method } \\
\cline { 2 - 3 } & $L_{\infty}$ & & & $L_{\infty}$ & $L_{2}$ \\
\hline 0.001 & $1.8221 \times 10^{-3}$ & $1.1556 \times 10^{-4}$ & & $7.4454 \times 10^{-8}$ & $5.2661 \times 10^{-8}$ \\
0.0005 & $5.6177 \times 10^{-4}$ & $2.9965 \times 10^{-5}$ & & $2.1479 \times 10^{-8}$ & $1.6911 \times 10^{-8}$ \\
0.00025 & $1.5380 \times 10^{-4}$ & $9.9200 \times 10^{-6}$ & & $5.4476 \times 10^{-9}$ & $5.1349 \times 10^{-9}$ \\
0.000125 & $3.9312 \times 10^{-5}$ & $1.9910 \times 10^{-6}$ & & $1.9831 \times 10^{-9}$ & $1.4734 \times 10^{-9}$ \\
\hline
\end{tabular}

Table 5 Error norms for Problem 2, when $N=80$ and $\Delta t=0.001$

\begin{tabular}{lllll}
\hline$\alpha$ & & $t=0.25$ & $t=0.50$ & $t=1$ \\
\hline 1.25 & $L_{\infty}$ & $2.8042 \times 10^{-9}$ & $5.0116 \times 10^{-9}$ & $7.8473 \times 10^{-9}$ \\
& $L_{2}$ & $1.8445 \times 10^{-9}$ & $3.4181 \times 10^{-9}$ & $5.3792 \times 10^{-9}$ \\
1.50 & $L_{\infty}$ & $2.4671 \times 10^{-8}$ & $3.8931 \times 10^{-8}$ & $7.4454 \times 10^{-8}$ \\
& $L_{2}$ & $1.4233 \times 10^{-8}$ & $2.7534 \times 10^{-8}$ & $5.2661 \times 10^{-8}$ \\
1.75 & $L_{\infty}$ & $2.1815 \times 10^{-8}$ & $6.7973 \times 10^{-8}$ & $1.7391 \times 10^{-8}$ \\
& $L_{2}$ & $1.4870 \times 10^{-8}$ & $4.7793 \times 10^{-8}$ & $1.2290 \times 10^{-7}$ \\
\hline
\end{tabular}

Table 6 Experimental order of convergence for Problem 2, when $N=80, \alpha=1.50, \Delta t=0.001$

\begin{tabular}{lllll}
\hline$N$ & $L_{\infty}$ & $E O C$ & $L_{2}$ & $E O C$ \\
\hline 10 & $4.2119 \times 10^{-4}$ & - & $1.6769 \times 10^{-4}$ & - \\
20 & $2.2034 \times 10^{-5}$ & 4.2566 & $9.9991 \times 10^{-6}$ & 4.0678 \\
40 & $1.8694 \times 10^{-6}$ & 4.1846 & $6.9693 \times 10^{-7}$ & 3.8452 \\
80 & $7.4454 \times 10^{-8}$ & 4.0245 & $5.2661 \times 10^{-8}$ & 3.8605 \\
\hline
\end{tabular}

The exact analytical solution to this problem is

$$
y(z, t)=\frac{1}{\pi^{5}}\left(\pi^{10} \sin (\pi z)+\cos (\pi z)-\cos (3 \pi z)\right)(t+1) .
$$

The computational error norms corresponding to four different selections of $\Delta t$ are given in Table 4 with $\gamma=0.05$ and $N=80$. It can be seen that our presented computational strategy yields more accurate solutions as compared to the technique used in [1]. Table 5 shows the maximum and Euclidian error norms at different time levels. The experimental rate of convergence is tabulated in Table 6 when error norms are calculated for $N=80, \alpha=1.50$, and $\Delta t=0.001$. It is clear that the experimental results support the theoretical estimation. Figure 5 displays the approximate solution at $t=1,2,3,4,5$. The three-dimensional plots of analytical exact and numerical solutions are portrayed in Fig. 6 in order to showcase their physical behaviour. In Fig. 7, the absolute computational error is presented at $t=1$ using $\alpha=1.50$ and $N=80$.

\section{Conclusion}

1. An algorithm utilizing quintic non-polynomial spline functions has been developed for the numerical treatment of time fractional fourth order superdiffusion equation.

2. The discretization in space and time directions has been achieved by using quintic non-polynomial spline functions and finite central difference formulation respectively.

3. The unconditional stability of the proposed scheme in temporal direction has been proved.

4. Theoretically, the presented technique is proved to be $O\left(h^{4}+\Delta t^{2}\right)$ accurate. 


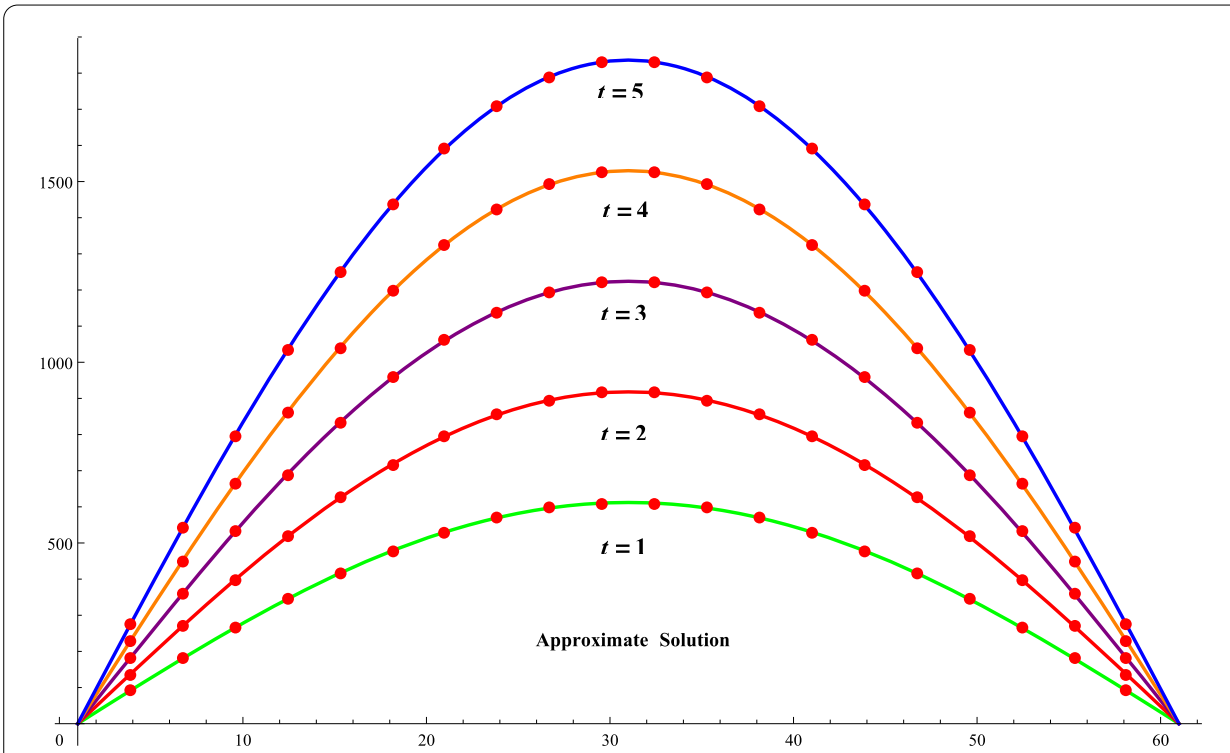

Figure 5 Approximate solution for Problem 2 at different time levels when $\Delta t=0.001, N=60, \alpha=1.25$

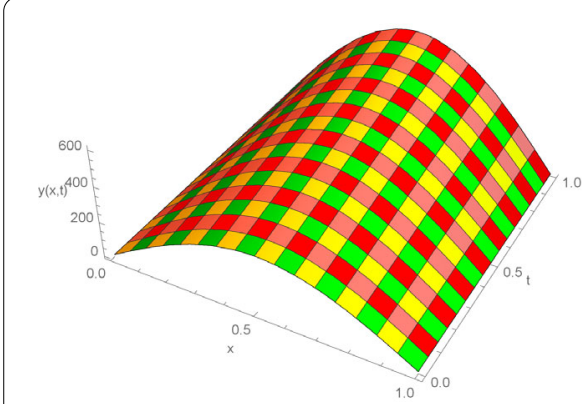

(a) Exact

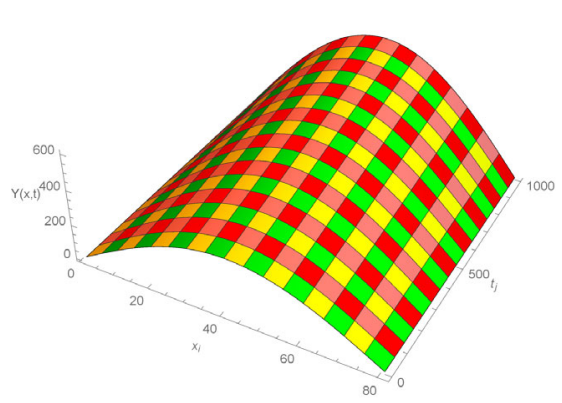

(b) Approximate

Figure 6 Exact and numerical solution for Problem 2 when $N=80, \alpha=1.25, \Delta t=0.001$

Figure 7 Absolute error for Problem 2 when $N=80$, $\Delta t=0.001$ and $\alpha=1.25$

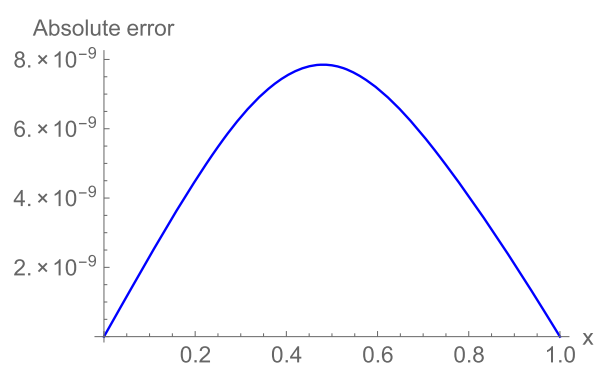

5. The experimental order of convergence is found to conform with the theoretical expectations.

6. The comparison of maximum and Euclidian error norms indicates the superiority of the present scheme over the method used in [1] even with larger grid spacing in time direction. 


\section{Acknowledgements}

The authors are also indebted to the anonymous reviewers for their helpful, valuable comments and suggestions in the improvement of this manuscript. This research was financial supported by Research University grant (1001/PMATHS/8011013) from School of Mathematical Sciences, Universiti Sains Malaysia.

\section{Funding}

All the financial support for publishing this paper is funded by Universiti Sains Malaysia.

\section{Availability of data and materials}

Not applicable.

\section{Competing interests}

The authors declare that they have no competing interests.

\section{Authors' contributions}

All authors equally contributed to this work. All authors read and approved the final manuscript.

\section{Authors' information}

Muhammad Amin is PhD scholar, Muhammad Abbas is Associate Professor, Muhammad Kashif Iqbal is Assistant Professor, Ahmad Izani Md. Ismail and Dumitru Baleanu are Professors.

\section{Author details}

'Department of Mathematics, National College of Business Administration \& Economics, Lahore, Pakistan. ${ }^{2}$ Department of Mathematics, University of Sargodha, Sargodha, Pakistan. ${ }^{3}$ Department of Mathematics, Government College University, Faisalabad, Pakistan. ${ }^{4}$ School of Mathematical Sciences, Universiti Sains Malaysia, Penang, Malaysia.

${ }^{5}$ Department of Mathematics, Faculty of Arts and Sciences, Cankaya University, Ankara, Turkey. ${ }^{6}$ Department of Medical Research, China Medical University Hospital, China Medical University, Taichung, Taiwan. ${ }^{7}$ Institute of Space Sciences, Magurele-Bucharest, Romania.

\section{Publisher's Note}

Springer Nature remains neutral with regard to jurisdictional claims in published maps and institutional affiliations.

\section{Received: 19 September 2019 Accepted: 2 December 2019 Published online: 12 December 2019}

\section{References}

1. Arshed, S:: Quintic b-spline method for time-fractional superdiffusion fourth-order differential equation. Math. Sci. 11(1), 17-26 (2017)

2. Tariq, H., Akram, G.: Quintic spline technique for time fractional fourth-order partial differential equation. Numer. Methods Partial Differ. Equ. 33(2), 445-466 (2017)

3. Podlubny, I.: Fractional Differential Equations: An Introduction to Fractional Derivatives, Fractional Differential Equations, to Methods of Their Solution and Some of Their Applications. Mathematics in Science and Engineering, vol. 198. Academic Press, San Diego (1998)

4. Miller, K.S., Ross, B.: An Introduction to the Fractional Calculus and Fractional Differential Equations. Wiley, New York (1993)

5. Jafari, H., Golbabai, A., Seifi, S., Sayevand, K.: Homotopy analysis method for solving multi-term linear and nonlinear diffusion-wave equations of fractional order. Comput. Math. Appl. 59(3), 1337-1344 (2010)

6. Dhaigude, D., Birajdar, G.A.: Numerical solution of system of fractional partial differential equations by discrete Adomian decomposition method. J. Fract. Calc. Appl. 3(12), 1-11 (2012)

7. El-Sayed, A., Gaber, M.: The Adomian decomposition method for solving partial differential equations of fractal order in finite domains. Phys. Lett. A 359(3), 175-182 (2006)

8. Momani, S., Odibat, Z.: Homotopy perturbation method for nonlinear partial differential equations of fractional order. Phys. Lett. A 365(5-6), 345-350 (2007)

9. Turut, V., Güzel, N.: On solving partial differential equations of fractional order by using the variational iteration method and multivariate Padé approximations. Eur. J. Pure Appl. Math. 6(2), 147-171 (2013)

10. Secer, A., Akinlar, M.A., Cevikel, A.: Efficient solutions of systems of fractional PDEs by the differential transform method. Adv. Differ. Equ. 2012(1), 188 (2012)

11. Zahra, W.K., Elkholy, S.M.: The use of cubic splines in the numerical solution of fractional differential equations. Int. J. Math. Math. Sci. 2012, Article ID 638026 (2012)

12. El Danaf, T.S.: Numerical solution for the linear time and space fractional diffusion equation. J. Vib. Control 21(9), 1769-1777 (2015)

13. Siddiqi, S.S., Arshed, S.: Numerical solution of time-fractional fourth-order partial differential equations. Int. J. Comput. Math. 92(7), 1496-1518 (2015)

14. Hamasalh, F.K., Muhammad, P.O.: Generalized quartic fractional spline interpolation with applications. Int. J. Open Problems Compt. Math. 8(1), 67-80 (2015)

15. Mohyud-Din, S.T., Akram, T., Abbas, M., Ismail, A.I., Ali, N.H.: A fully implicit finite difference scheme based on extended cubic b-splines for time fractional advection-diffusion equation. Adv. Differ. Equ. 2018(1), 109 (2018)

16. Li, M., Ding, X., Xu, Q.: Non-polynomial spline method for the time-fractional nonlinear Schrödinger equation. Adv. Differ. Equ. 2018(1), 318 (2018)

17. Pezza, L., Pitolli, F.: A fractional spline collocation-Galerkin method for the time-fractional diffusion equation. Commun. Appl. Ind. Math. 9(1), 104-120 (2018) 
18. Khalid, N., Abbas, M., lqbal, M.K.: Non-polynomial quintic spline for solving fourth-order fractional boundary value problems involving product terms. Appl. Math. Comput. 349, 393-407 (2019)

19. Amin, M., Abbas, M., Iqbal, M.K., Baleanu, D.: Non-polynomial quintic spline for numerical solution of fourth-order time fractional partial differential equations. Adv. Differ. Equ. 2019(1), 183 (2019)

20. Khalid, N., Abbas, M., Iqbal, M.K., Baleanu, D.: A numerical algorithm based on modified extended B-spline functions for solving time-fractional diffusion wave equation involving reaction and damping terms. Adv. Differ. Equ. 2019, 378 (2019)

21. Lin, Y., Xu, C.: Finite difference/spectral approximations for the time-fractional diffusion equation. J. Comput. Phys. 225(2), 1533-1552 (2007)

22. Iqbal, M.K., Abbas, M., Wasim, l.: New cubic b-spline approximation for solving third order Emden-Flower type equations. Appl. Math. Comput. 331, 319-333 (2018)

23. Wasim, I., Abbas, M., Amin, M.: Hybrid b-spline collocation method for solving the generalized Burgers-Fisher and Burgers-Huxley equations. Math. Probl. Eng. 2018, Article ID 6143934 (2018)

\section{Submit your manuscript to a SpringerOpen ${ }^{\circ}$} journal and benefit from:

- Convenient online submission

- Rigorous peer review

Open access: articles freely available online

- High visibility within the field

- Retaining the copyright to your article

Submit your next manuscript at $\gg$ springeropen.com 